\title{
Excited-state Koopmans theorem for ensembles
}

\author{
M. Levy ${ }^{1}$ and Á. Nagy ${ }^{2}$ \\ ${ }^{1}$ Department of Chemistry and Quantum Theory Group, Tulane University, New Orleans, Louisiana 70118 \\ ${ }^{2}$ Institute of Theoretical Physics, Kossuth Lajos University, H-4010 Debrecen, Hungary
}

(Received 20 March 1998; revised manuscript received 15 October 1998)

\begin{abstract}
Koopmans's theorem is generalized for excited-state ensemble density-functional theory. Formal expressions are derived that relate orbital energy differences to exchange-only excitation energies. These expressions provide stringent requirements for approximating the universal exchange component of the exact exchangecorrelation functional for excited-state ensembles, and the expressions lead to a relation, containing a correlation potential, that gives exact excited-state ionization energies. [S1050-2947(99)03202-3]
\end{abstract}

PACS number(s): 31.15.Ew

\section{INTRODUCTION}

In ensemble density-functional theory for excited states [1-3], there is an unknown exchange-correlation functional that must be approximated. For this purpose, it is necessary to invoke known properties of the exact functional. With this in mind, we here derive a relation that connects the repulsion energies of the components of the auxiliary noninteracting Kohn-Sham ensemble with an integral containing the corresponding ensemble repulsion functional derivative. This relation, Eq. (28), is one that involves the exchange component of the unknown exchange-correlation functional. Further, when this relation is combined with an earlier one [4] involving the ensemble correlation potential, $v_{c}$, a new relation results, Eq. (29), that connects the exact excited-state ionization energy with expectation values involving noninteracting Kohn-Sham wave-function solutions and $v_{c}$. Finally, manipulation of Eq. (28) allows the development of an exchange-only excited-state Koopmans theorem, Eq. (9), that relates, with a small correction term, an orbital energy difference, involving an unoccupied orbital from an exchangeonly ground-state calculation, to an expectation value difference involving Kohn-Sham determinants and the true physical Hamiltonian of interest.

\section{THEOREM}

Let $n_{\mathrm{GS}}$ be the exchange-only ground-state density of the $N$-electron Hamiltonian

$$
\hat{H}_{v}=\hat{T}+\hat{V}_{e e}+\sum_{i=1}^{N} v\left(r_{i}\right)
$$

where $\hat{T}$ is the kinetic energy operator and $\hat{V}_{e e}$ is the electron-electron repulsion energy operator. The auxiliary Kohn-Sham equations for $n_{\mathrm{GS}}$ are

$$
\left\{-\frac{1}{2} \nabla^{2}+v_{s}\left(r ;\left[n_{\mathrm{GS}}\right]\right)\right\} \phi_{i}(r)=\epsilon_{i} \phi_{i}(r)
$$

where

$$
\epsilon_{0} \leqslant \epsilon_{1} \leqslant \epsilon_{2} \cdots
$$

and
Let us consider, for simplicity of presentation, the closedshell case, for even $N$, where

$$
n_{\mathrm{GS}}=\sum_{i=1}^{N / 2} 2\left|\phi_{i}\right|^{2}
$$

Call $\Phi_{\mathrm{GS}}$, whose density is $n_{\mathrm{GS}}$, the closed-shell determinant which is composed of the first $N / 2$ doubly occupied Kohn-Sham $\Phi_{i}$. In other words, $\Phi_{\mathrm{GS}}$ is the ground state of some local potential $v_{s}$ and simultaneously minimizes the expectation values of $\hat{H}_{v}$ in Eq. (1). That is, $\Phi_{\mathrm{GS}}$ is the ground state of the noninteracting Kohn-Sham Hamiltonian

$$
\hat{H}_{s}=\sum_{i=1}^{N}-\frac{1}{2} \nabla_{i}^{2}+\sum_{i=1}^{N} v_{s}\left(r ;\left[n_{\mathrm{GS}}\right]\right) .
$$

Next, define $\Phi_{\mathrm{ES}}^{k}$ as an excited state of $\hat{H}_{s}$ that is formed from $\Phi_{\mathrm{GS}}$ by exciting an electron from orbital $i=N / 2$ to orbital $i=k$. The density corresponding to $\Phi_{\mathrm{ES}}^{k}$ has the form

$$
n_{\mathrm{ES}}^{k}=\sum_{i=1}^{M} 2\left|\phi_{i}\right|^{2}+\left|\phi_{N / 2}\right|^{2}+\left|\phi_{k}\right|^{2} \text {, }
$$

where

$$
M=(N / 2)-1
$$

Consider the ensemble density $n$ which is composed of a sum such that each element in the sum consists of a nonzero weight factor times an eigenstate density of $\hat{H}_{s}[1-3]$. It is understood that included in the sum are all densities whose energies, with respect to $\hat{H}_{s}$, are less than or equal to the energy of $n_{\mathrm{ES}}^{k}$. With $n$ so defined, we shall prove the following ensemble Koopmans theorem for excited states:

$$
\begin{aligned}
\epsilon_{k} & -\epsilon_{N / 2}+\int \Delta v(r)\left|\phi_{k}(r)\right|^{2} d r \\
& =\left\langle\Phi_{\mathrm{ES}}^{k}\left|\hat{H}_{v}\right| \Phi_{\mathrm{ES}}^{k}\right\rangle-\left\langle\Phi_{\mathrm{GS}}\left|\hat{H}_{v}\right| \Phi_{\mathrm{GS}}\right\rangle,
\end{aligned}
$$


where $\Delta v(\mathbf{r})=v(\mathbf{r})-v^{\prime}(\mathbf{r})$, and $v^{\prime}$ is that external potential for which $n$ is the optimum Kohn-Sham ensemble density for an exchange-only ensemble calculation, and where $\Phi_{\mathrm{ES}}^{k}$ is an excited state of $\hat{H}_{s}$ that yields $n_{\mathrm{ES}}^{k}$. The potential $v$, on the other hand, is that external potential in Hamiltonian (1) for which $n_{\mathrm{GS}}$ is the optimum Kohn-Sham ground-state density in the exchange-only approximation. Also in Eq. (9)

$$
\epsilon_{N / 2}=\left\langle\phi_{N / 2}\left|-\frac{1}{2} \nabla^{2}+v_{s}\left(r ;\left[n_{\mathrm{GS}}\right]\right)\right| \phi_{N / 2}\right\rangle
$$

and

$$
\epsilon_{k}=\left\langle\phi_{k}\left|-\frac{1}{2} \nabla^{2}+v_{s}\left(r ;\left[n_{\mathrm{GS}}\right]\right)\right| \phi_{k}\right\rangle,
$$

where $\varphi_{N / 2}$ is the highest-occupied orbital in the ground-state exchange-only calculation.

\section{PROOF OF THE THEOREM}

In line with the definition of $v^{\prime}$, we have

$$
\min _{\rho}\left\{\int v^{\prime}(r) \rho(r) d r+F_{\mathrm{xo}}[\rho]\right\}=\int v^{\prime}(r) n(r) d r+F_{\text {хо }}[n],
$$

where $\rho(\mathbf{r})$ is an arbitrary ensemble density given by

$$
\rho(r)=\operatorname{tr}\{\hat{D} \hat{\rho}(r)\},
$$

where $\hat{\rho}(r)$ is the density operator. The noninteracting density matrix $\hat{D}$ is defined as

$$
\hat{D}=\sum_{i=1}^{k} w_{i}\left|\Phi_{i}\right\rangle\left\langle\Phi_{i}\right|
$$

while the exchange-only functional $F_{\text {xo }}$ has the definition

$$
F_{\text {xo }}=\operatorname{tr}\left\{\hat{D}\left(\hat{T}+\hat{V}_{e e}\right)\right\},
$$

where the $\Phi_{i}$ in Eq. (14) yield $\rho$ through Eq. (13) and are simultaneously the lowest eigenfunctions of some noninteracting Hamiltonian, and the weights $w_{i}$ are understood to be the same as those with $n$. The minimization in Eq. (12) dictates that the potential $v^{\prime}$ can be expressed as

$$
v^{\prime}=-\left.\frac{\delta F_{\mathrm{xo}}[\rho]}{\delta \rho}\right|_{\rho=n}=v_{s}-\left.\frac{\delta V_{e e}^{\mathrm{KS}}[\rho]}{\delta \rho}\right|_{\rho=n},
$$

where

$$
V_{e e}^{\mathrm{KS}}[\rho]=\operatorname{tr}\left\{\hat{D} \hat{V}_{e e}\right\} .
$$

In Eq. (16) it is understood, for later development, that $v_{s}$ and $\delta V_{e e}^{\mathrm{KS}}[\rho] / \delta \rho$ have been made to vanish as $|\mathbf{r}| \rightarrow \infty$, through additive constants as necessary.

Next, let $n$ be the optimum density for

$$
\hat{H}_{\alpha}=\hat{T}+\alpha \hat{V}_{e e}+\sum_{i=1}^{N} g_{\alpha}\left(r_{i} ;[n]\right) .
$$

In other words, $g_{\alpha}\left(\mathbf{r}_{i} ;[n]\right)$ is constructed such that the ensemble density $n$, with fixed weights, remains independent of $\alpha$. Note that $g_{0}=v_{s}$ where $v_{s}$ is the Kohn-Sham potential in Eq. (2). In other words, $\hat{H}_{0}=\hat{H}_{s}$. However, $g_{1}$ is neither $v$ nor $v^{\prime}$, although close to both. Define $n_{\mathrm{GS}}^{\alpha}$ and $E_{\mathrm{GS}}^{\alpha}$, respectively, as the ground-state density and energy of $\hat{H}_{\alpha}$, and define the eigenstate density $n_{\mathrm{ES}}^{k, \alpha}$ as that ensemble component of $n$ with the highest energy, $E_{\mathrm{ES}}^{k, \alpha}$, with respect to $\hat{H}_{\alpha}$. Then, in accordance with previous studies [5], the asymptotic decay of $n_{\mathrm{GS}}$ is governed by $\left|E_{\mathrm{GS}}^{\alpha}-E_{\mathrm{GS}}^{\alpha, N-1}\right|$, while unless prohibited by symmetry, the asymptotic decay of $n_{\mathrm{ES}}^{k, \alpha}$ is governed by $\left|E_{\mathrm{ES}}^{k, \alpha}-E_{\mathrm{GS}}^{\alpha, N-1}\right|$, where $E_{\mathrm{GS}}^{\alpha, N-1}$ is the ground-state energy of $\hat{H}_{\alpha}$ with one electron removed. Consequently [4], the asymptotic decay of $n$ is governed by $\left|E_{\mathrm{ES}}^{k, \alpha}-E_{\mathrm{GS}}^{\alpha, N-1}\right|$. This means that $\left|E_{\mathrm{ES}}^{k, \alpha}-E_{\mathrm{GS}}^{\alpha, N-1}\right|$ is independent of $\alpha$ because $n$ is independent of $\alpha$. (Observe that $n_{\mathrm{ES}}^{k, \alpha}$ connects with $n_{\mathrm{ES}}^{k}$ as $\alpha \rightarrow 0$.)

Next, following the procedure of Ref. [6] for a groundstate density-functional theory (DFT) Koopmans theorem, we here employ

$$
\left.\frac{\partial}{\partial \alpha}\left(E_{\mathrm{ES}}^{k, \alpha}-E_{\mathrm{GS}}^{\alpha, N-1}\right)\right|_{\alpha=0}=0,
$$

and using Eq. (18), we obtain

$$
\begin{gathered}
\left\langle\Phi_{\mathrm{ES}}^{k}\left|\hat{V}_{e e}\right| \Phi_{\mathrm{ES}}^{k}\right\rangle+\left.\int d r n_{\mathrm{ES}}^{k} \frac{\partial g_{\alpha}}{\partial \alpha}\right|_{\alpha=0} \\
=\left\langle\Phi_{\mathrm{GS}}^{N-1}\left|\hat{V}_{e e}\right| \Phi_{\mathrm{GS}}^{N-1}\right\rangle+\left.\int d r n_{\mathrm{GS}}^{N-1} \frac{\partial g_{\alpha}}{\partial \alpha}\right|_{\alpha=0},
\end{gathered}
$$

where $\Phi_{\mathrm{GS}}^{N-1}$ and $n_{\mathrm{GS}}^{N-1}$ are, respectively, the ground-state wave function and density of the Kohn-Sham $\hat{H}_{s}$ of Eq. (6), with one electron removed.

To identify the partial derivative in Eq. (20) we observe that from the analogous ground-state adiabatic connection expansion [7], it follows here, for small $\alpha$, that

$$
g_{\alpha}(r ;[n])=v_{s}-\left.\alpha \frac{\delta V_{e e}^{\mathrm{KS}}[\rho]}{\delta \rho}\right|_{\rho=n}+\cdots
$$

This leads to

$$
\left.\frac{\partial g_{\alpha}}{\partial \alpha}\right|_{\alpha=0}=-\left.\frac{\delta V_{e e}^{\mathrm{KS}}}{\delta \rho}\right|_{\rho=n} .
$$

Comparison with Eq. (16) gives

$$
\left.\frac{\partial g_{\alpha}}{\partial \alpha}\right|_{\alpha=0}=v^{\prime}(r)-v_{s}(r,[n])
$$

and the substitution of Eq. (23) into Eq. (20) leads to

$$
\epsilon_{k}=\left\langle\Phi_{\mathrm{ES}}^{k}\left|\hat{H}_{v^{\prime}}\right| \Phi_{\mathrm{ES}}^{k}\right\rangle-\left\langle\Phi_{\mathrm{GS}}^{N-1}\left|\hat{H}_{v^{\prime}}^{N-1}\right| \Phi_{\mathrm{GS}}^{N-1}\right\rangle,
$$

where $\hat{H}_{v^{\prime}}^{N-1}$ is $\hat{H}_{v^{\prime}}$ with one electron removed. Equation (24) may be conveniently written as 


$$
\begin{aligned}
\epsilon_{k}+\int \Delta v(r)\left|\phi_{K}(r)\right|^{2}= & \left\langle\Phi_{\mathrm{ES}}^{k}\left|\hat{H}_{v}\right| \Phi_{\mathrm{ES}}^{k}\right\rangle \\
& -\left\langle\Phi_{\mathrm{GS}}^{N-1}\left|\hat{H}_{v}^{N-1}\right| \Phi_{\mathrm{GS}}^{N-1}\right\rangle,
\end{aligned}
$$

where $\hat{H}_{v}^{N-1}$ is $\hat{H}_{v}$ with one electron removed.

Next, we use the following ground-state Koopmans theorem, which was previously arrived at for finite $[8,6]$ and infinite systems [9]:

$$
\epsilon_{N / 2}=\left\langle\Phi_{\mathrm{GS}}\left|\hat{H}_{v}\right| \Phi_{\mathrm{GS}}\right\rangle-\left\langle\Phi_{\mathrm{GS}}^{N-1}\left|\hat{H}_{v}\right| \Phi_{\mathrm{GS}}^{N-1}\right\rangle .
$$

The combination of Eqs. (25) and (26) yields our first desired result, Eq. (9). Further, combine Eqs. (20) and (22) to obtain

$$
\begin{gathered}
\left\langle\Phi_{\mathrm{ES}}^{k}\left|\hat{V}_{e e}\right| \Phi_{\mathrm{ES}}^{k}\right\rangle-\left\langle\phi_{\mathrm{GS}}^{N-1}\left|\hat{V}_{e e}\right| \Phi_{\mathrm{GS}}^{N-1}\right\rangle \\
=\left.\int d r\left(n_{\mathrm{ES}}^{k}-n_{\mathrm{GS}}^{N-1}\right) \frac{\delta V_{e e}^{\mathrm{KS}}}{\delta \rho}\right|_{\rho=n},
\end{gathered}
$$

or

$$
\begin{gathered}
\left\langle\Phi_{\mathrm{ES}}^{k}\left|\hat{V}_{e e}\right| \Phi_{\mathrm{ES}}^{k}\right\rangle-\left\langle\Phi_{\mathrm{GS}}^{N-1}\left|\hat{V}_{e e}\right| \Phi_{\mathrm{GS}}^{N-1}\right\rangle \\
=\left.\int d r\left|\phi_{k}(r)\right|^{2} \frac{\delta V_{e e}^{\mathrm{KS}}}{\delta \rho}\right|_{\rho=n} .
\end{gathered}
$$

Equation (28) is in the form which is especially useful for imposing a stringent constraint for approximating $V_{e e}^{\mathrm{KS}}[\rho]$.

Finally, observe that by Eq. (24) and by the fact $[4,5]$ that $\epsilon_{k}=E_{\mathrm{ES}}^{k, 1}-E_{\mathrm{GS}}^{1, N-1}$, it follows with $\alpha=1$ in Eq. (18) that

$$
\begin{aligned}
E_{\mathrm{ES}}^{k, 1}-E_{\mathrm{GS}}^{1, N-1}= & \left\langle\Phi_{\mathrm{ES}}^{k}\left|\hat{H}_{1}+\sum_{i=1}^{N} v_{c}\left(\vec{r}_{i}\right)\right| \Phi_{\mathrm{ES}}^{k}\right\rangle \\
& -\left\langle\Phi_{\mathrm{GS}}^{N-1}\left|\hat{H}_{1}^{N-1}+\sum_{i=1}^{N-1} v_{c}\left(\vec{r}_{i}\right)\right| \Phi_{\mathrm{GS}}^{N-1}\right\rangle,
\end{aligned}
$$

where $\hat{H}_{1}^{N-1}$ is the $\hat{H}_{1}$ in Eq. (18) with one electron removed, and where $v_{c^{\prime}}$ which equals $v^{\prime}-g_{1}$, is the ensemble correlation potential for $n$. (This correlation potential identi- fication for $v^{\prime}-g$ makes sense because $g_{1}$ is that potential for which $n$ is a sum of eigenstate densities, obtained when correlation is included, while $v^{\prime}$ is that potential for which $n$ is the optimum ensemble density for an exchange-only excited-state ensemble calculation, where by definition, correlation is excluded.). Equation (29) gives the exact ionization energy from the highest eigenstate of $\hat{H}_{1}$ whose density is a component of $n$. Of special relevance, of course, is when $g_{1}$ is the atomic, molecular, or solid-state external potential of interest.

\section{CONCLUDING REMARKS}

Constraints (9), (27), and (28) serve as stringent requirements for the exchange component of the exchangecorrelation functional for excited-state ensemble calculations. Further, observe that analogous constraints arise with any excited-state formulation for which Eq. (19) applies.

It was previously observed [4] that the negative of the highest-occupied Kohn-Sham orbital energy of the noninteracting auxiliary ensemble gives the exact ionization energy, including correlation, of the highest-energy eigenstate in the occupied interacting ensemble for the true physical interacting Hamiltonian of interest. In the present paper we have shown that Eq. (29) provides an alternative formula for this exact eigenstate ionization energy from an ensemble calculation.

Finally, the ensemble Koopmans theorem for excited states, Eq. (9), is of special relevance for the study of the use of unoccupied orbital energies in the approximation of excitation energies. In this connection, it is worth noting the fact that cases have recently been reported, with explanation, where these unoccupied orbital energies give surprisingly accurate excited-state ionization energies. (See Ref. [10], and references therein.)

\section{ACKNOWLEDGMENTS}

This work was supported by the MTA-NSF under Grant No. 93, OTKA under Grant No. T 16623, and FKFP under Grant No. 0314/1997. Financial support from the U.S. National Institute of Standards and Technology, as well as the "Széchenyi" grant from the Hungarian Ministry of Culture and Education are gratefully acknowledged.
[1] A. K. Theophilou, J. Phys. C 12, 5419 (1978).

[2] E. K. U. Gross, L. N. Oliveira, and W. Kohn, Phys. Rev. A 37, 2805 (1988); 37, 2809 (1988); 37, 2821 (1988).

[3] Á. Nagy, Phys. Rev. A 42, 4388 (1990): Int. J. Quantum Chem. 56, 225 (1995); Int. J. Quantum Chem., Symp. 29, 297 (1995): Adv. Quantum Chem. 29, 159 (1997).

[4] M. Levy, Phys. Rev. A 52, R4313 (1995).

[5] For a very recent review see J. P. Perdew and M. Levy, Phys. Rev. B 56, 16021 (1997).

[6] M. Levy and A. Görling, Phys. Rev. A 53, 3140 (1996).

[7] A. Görling and M. Levy, Phys. Rev. B 47, 13105 (1993);
Phys. Rev. A 50, 196 (1994); Int. J. Quantum Chem., Symp. 29, 93 (1995).

[8] J. B. Krieger, Y. Li, and G. J. Iafrate, Phys. Rev. A 45, 101 (1992); 46, 5453 (1992).

[9] J. P. Perdew, in Density Functional Methods in Physics, Vol. 337 of NATO Advanced Study Institute, Series B: Physics, edited by R. M. Dreizler and J. de Providencia (Plenum, New York, 1995).

[10] C. Filippi, C. J. Umrigar, and X. Gonze, J. Chem. Phys. 107, 9994 (1997). 